\title{
Longévité des graines et contraintes à la survie des plantules d'Afzelia africana Sm. dans une savane boisée du Burkina Faso
}

\author{
Babou André Bationo, ${ }^{\mathrm{a},}$, Sibiri Jean Ouedraogo ${ }^{\mathrm{a}}$ et Sita Guinko ${ }^{\mathrm{b}}$ \\ a Institut de l'Environnement et de Recherches Agricoles, Département Productions Forestières (I.N.E.R.A./D.P.F.), \\ 03 BP 7047 Ouagadougou 03, Burkina Faso \\ ${ }^{\mathrm{b}}$ Faculté des Sciences et Techniques, Laboratoire de Biologie et Écologie végétale, BP 7021 Ouagadougou, Burkina Faso
}

(Reçu le 13 juin 2000; accepté le 25 août 2000)

\begin{abstract}
Résumé - La viabilité des semences et la capacité de survie des plantules sont des déterminants essentiels de la régénération séminale des espèces végétales en milieu naturel. Des études sur les contraintes à la régénération séminale d'Afzelia africana ont été conduites au laboratoire, en pépinière et en milieu naturel. Elles ont consisté en des tests de germination et, au suivi de la croissance des plantules en rhizotron et en analyse des facteurs de mortalité en milieu naturel. Les résultats ont révélé que le maintien de la viabilité des graines ne nécessite pas des précautions particulières de conservation. La teneur initiale en eau des graines était de l'ordre de $8 \%$ (rapporté au poids frais), et ont été conservées dans les conditions ambiantes pendant au moins 33 mois après la récolte sans que cela n'ait affecté significativement le taux de germination. Les plantules ont présenté un système racinaire pivotant et précocement traçant dans le rhizotron. Sur le terrain, nous avons noté une très forte mortalité due à leur sensibilité aux feux, au broutage et à la sécheresse. La réussite du semis direct d’A. africana nécessite donc une protection contre ces facteurs.
\end{abstract}

\section{Afzelia africana / régénération / rhizotron / savane / Burkina Faso}

\begin{abstract}
The longevity of seed and the constraints of survival of seedlings of Afzelia africana Sm. in a woody savannah in Burkina Faso. The viability of seeds and the survival of seedlings in natural conditions are key factors for sexual regeneration of woody species. The constraints of sexual regeneration of Afzelia africana were studied in laboratory, nursery and under natural conditions. The experiments consisted of germination tests in laboratory, monitoring of the seedling growth in rhizotron and assessment of the factors of mortality of seedlings under natural conditions. When seeds water content is about $8 \%$ (on the fresh weight basis), they can be stored in ambient conditions for at least 33 months after collection, without a significant reduction of the germination rate. The seedlings have a deep-root system with precocious lateral ramifications. However, the seedlings are still very sensitive to fire, browsing and drought. To grow well, the seedlings of $A$. africana need to be protected against these constraints.
\end{abstract}

Afzelia africana / regeneration / rhizotron / savannah / Burkina Faso

\section{INTRODUCTION}

La longévité des semences et la survie des plantules sont des facteurs déterminants de la régénération séminale des espèces forestières [2]. La viabilité des semences et la résistance des plantules aux multiples agressions en milieu naturel peuvent être déterminées par des facteurs biologiques et écophysiologiques propres aux espèces elles-mêmes [5, 13, 14, 15, 23]. C'est le cas par exemple de la teneur en eau des graines qui affecte la longévité et les conditions de conservation des semences [17]. De même, la morphologie fonctionnelle des plantules

* Correspondance et tirés-à-part

Tél. (226) 3340 98; Fax. (226) 314938. 
conditionne de façon significative l'adaptabilité de celles-ci aux facteurs souvent très contraignants du milieu naturel [7, 18, 20, 22].

Afzelia africana est l'une des espèces de valeur au Burkina Faso. Elle est utilisée dans l'alimentation humaine et animale, et comme bois d'œuvre. Depuis près d'une décennie l'on a recours à la technique du semis direct pour pallier sa mauvaise régénération dans les formations naturelles. Cependant, depuis l'adoption de cette technique de régénération, aucune étude n'a évalué son impact sur la régénération des parcelles ensemencées. Ces parcelles sont par ailleurs parcourues par les feux précoces chaque année et constamment pâturées par le bétail sans que l'on ne dispose des connaissances sur les capacités de résistance des plantules d'A. africana à ces facteurs. De même, les connaissances sur l'influence des conditions de conservation en milieu paysan sur la viabilité des semences sont presque inexistantes. Ceci limite l'appropriation de la gestion des stocks de semences par les populations rurales elles-mêmes. C'est dans ce contexte qu'il nous a paru nécessaire d'appréhender l'influence sur la longévité des graines d'A. africana de la durée de conservation dans les conditions ambiantes et d'évaluer l'impact des ensemencements antérieurs sur le recrutement de l'espèce ainsi que les capacités d'adaptation des plantules aux agressions récurrentes du milieu. Les résultats de cette étude devraient à terme dégager la possibilité d'une gestion des semences en milieu rural et les capacités du semis direct à pallier la médiocrité de la régénération naturelle.

\section{MATÉRIEL ET MÉTHODES D’ÉTUDE}

\subsection{Site d'étude}

L'étude a été conduite dans une savane boisée appelée «forêt classée de Nazinon», à $100 \mathrm{~km}$ au sud du Burkina Faso. Le climat est de type soudanien [9] avec une seule saison pluvieuse de mai à octobre. La pluviométrie moyenne annuelle est de l'ordre de 800 à $900 \mathrm{~mm}$ et dépasse rarement $1000 \mathrm{~mm}$. Les mois les plus chauds sont mars et avril où les températures moyennes journalières varient entre 30 et $35{ }^{\circ} \mathrm{C}$. Les mois les plus froids sont décembre et janvier avec des températures oscillant entre 20 et $27{ }^{\circ} \mathrm{C}$ [3]. Les données pédologiques établissent une prédominance de sols ferrugineux tropicaux lessivés indurés, avec généralement une carapace entre 50 et $100 \mathrm{~cm}$ de profondeur [4]. Depuis 1989, la forêt classée est soumise à un plan d'aménagement dont l'objectif principal est la production de bois de chauffe pour approvisionner la ville de Ouagadougou. L'aménagement est caractérisé principalement par la pratique des feux précoces et l'ensemencement par semis direct des parcelles exploitées.

\section{Germination des graines, morphologie \\ fonctionnelle, croissance et survie des plantules}

En 1997 nous avons récolté sous les semenciers un lot de graines d'Afzelia africana. Un échantillon de 100 graines a été aussitôt prélevé et le poids frais (pf) individuel a été enregistré à l'aide d'une balance de sensibilité $10^{-3} \mathrm{~g}$. Cet échantillon a été passé à l'étuve à $60^{\circ} \mathrm{C}$ pendant une semaine, période au bout de laquelle un poids constant a été obtenu, et le poids sec (ps) de chaque graine a été mesuré. La teneur en eau par rapport au poids frais et au poids sec a été ensuite calculée respectivement par les formules suivantes utilisées par Willan (1992) : $100 \times(\mathrm{pf}-\mathrm{ps}) / \mathrm{pf}$ et $100 \times(\mathrm{pf}-\mathrm{ps}) / \mathrm{ps}$. Les graines restantes ont été ensuite conservées en sac dans une armoire en bois. Après 1,15 et 33 mois de stockage un échantillon de 100 graines a été prélevé et mis à germer sans prétraitement, dans des boîtes de pétri sur du papier filtre.

La croissance et la morphologie des plantules ont été suivies en pépinière dans un rhizotron permettant un suivi continu de la croissance souterraine des plantules. Nous avons utilisé un rhizotron rectangulaire à cadre en bois avec une face amovible. Sa hauteur était d'un mètre avec une contenance de $0,15 \mathrm{~m}^{3}(0,3 \times 0,5 \times 1 \mathrm{~m})$. Une vitre claire et transparente d'une épaisseur de $8 \mathrm{~cm}$ et inclinée de $45{ }^{\circ} \mathrm{C}$ par rapport à la verticale, subdivisait le rhizotron en deux compartiments. L'un était rempli de sol. L'autre, du côté de la face amovible, était vide et permettait d'accéder à la vitre pour observer régulièrement le développement des racines.

Le profil pédologique dans le rhizotron a été constitué à partir du sol des horizons du profil dominant dans la zone d'étude. Il comportait quatre horizons $\left(\mathrm{H}_{1}, \mathrm{H}_{2}, \mathrm{H}_{3}\right.$ et $\mathrm{H}_{4}$ ). La granulométrie de chaque horizon a été déterminée au laboratoire à partir d'un échantillon composite (tableau I). Pendant le remplissage du rhizotron le sol était damé pour être proche des conditions naturelles. Quatre graines ont été ensuite semées 1 à $2 \mathrm{~cm}$ de la vitre. Cela permettait à la radicule d'être visible à travers la vitre dès son émergence. Les apports en eau après la

Tableau I. Caractéristiques du profil pédologique dans le rhizotron.

\begin{tabular}{lcccc}
\hline & $\mathrm{H}_{1}$ & $\mathrm{H}_{2}$ & $\mathrm{H}_{3}$ & $\mathrm{H}_{4}$ \\
\hline Sable total (\%) & 69,29 & 66,50 & 55,38 & 40,89 \\
Argile totale (\%) & 5,75 & 11,75 & 28,25 & 37 \\
Limon total (\%) & 24,96 & 21,75 & 16,37 & 22,11 \\
Epaisseur (cm) & 15 & 20 & 20 & 40 \\
\hline
\end{tabular}


germination étaient assurés par les pluies. Pendant trois mois et demi (début-juillet à mi-octobre), la croissance du pivot et de la tige principale de chaque plantule a été enregistrée à une fréquence hebdomadaire. Les observations sur le système racinaire ont été faites après la tombée du jour à l'aide d'une ampoule inactinique afin d'éviter les effets de la lumière blanche sur les racines. Les accroissements hebdomadaires du pivot étaient matérialisés sur la vitre par des tracés de couleurs différentes. Les études en milieu semi-contrôlé ont été complétées par des observations sur le développement des racines de plus de 20 jeunes plantules en fonction de la structure du sol en milieu naturel.

Afin d'évaluer l'impact du semis direct sur la régénération d'Afzelia africana dans la forêt, un inventaire des plantules a été effectué en septembre 1997 sur les parcelles ensemencées en 1992, 1993, 1994, 1995 et 1996. Sur chaque parcelle l'inventaire a été réalisé le long de deux transects de $10 \mathrm{~m}$ de large suivant respectivement la longueur et la largeur de la parcelle. La longueur des transects variait de 3,5 à $6 \mathrm{~km}$.

Enfin, la levée des graines après le semis direct, la capacité des jeunes plantules à survivre à l'installation de la sécheresse et surtout aux feux précoces ont été suivies de septembre 1998 à janvier 1999. Les observations ont été faites sur la parcelle ensemencée par les bûcherons en juillet 1998 après l'exploitation. Le semis direct s'effectue suivant des lignes. Généralement deux ou trois graines sont semées par poquet. L'écartement est de $4 \mathrm{~m}$ entre les lignes et entre les poquets sur une même ligne. Sur la parcelle, deux transects de $1 \mathrm{~km}$ chacun et perpendiculaires en leur centre ont été identifiés. Le long de chaque transect, des placeaux de $10 \mathrm{~m}^{2}$ pouvant contenir au maximum 9 poquets chacun, ont été installés à intervalles réguliers de $100 \mathrm{~m}$. À l'intérieur de chaque placeau le nombre de poquets avec au moins une plantule et le nombre de plantules dans chaque poquet «fertile» ont été enregistré en début septembre. Les plantules vivantes ont été ensuite inventoriées un mois et demi après la saison pluvieuse, puis un mois après le passage des feux précoces. Les résultats ont été analysés à l'aide du logiciel STATITCF, au seuil de $5 \%$.

\section{RESULTATS}

La teneur en eau des graines a été de 8,33\% et 9,15\% respectivement par rapport au poids frais et au poids sec. La germination s'est étalée sur une période d'environ un mois. Les graines fraîchement récoltées présentaient eux un taux de germination supérieur à $90 \%$. Ce taux atteignait toujours $80 \% 33$ mois après la récolte (figure 1 ). Les observations en rhizotron révèlent une germination épigée et non cryptogée des graines. La figure 2 montre

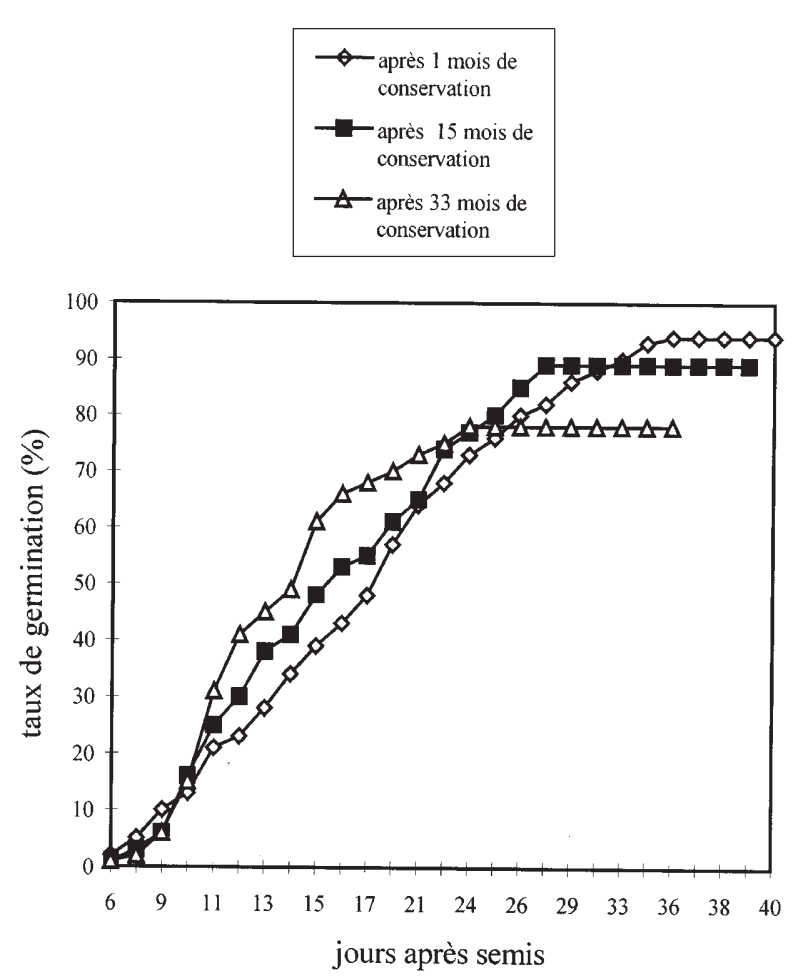

Figure 1. Influence de la durée de conservation dans les conditions ambiantes sur la germination de graines d'Afzelia africana.

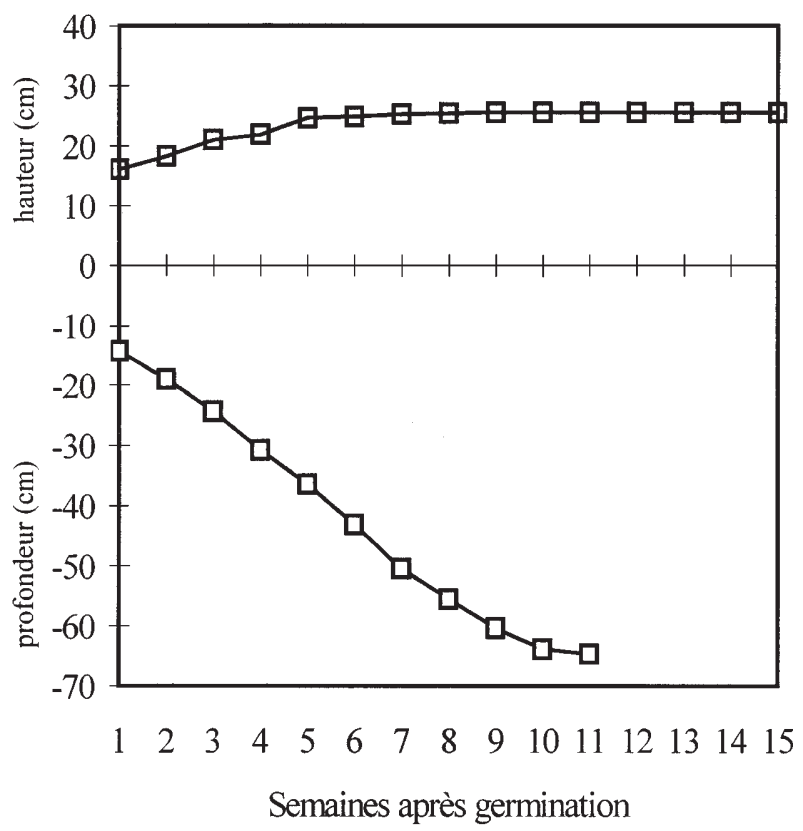

Figure 2. Croissance hebdomadaire cumulée de la tige principale et du pivot de plantules d'A. africana élevées en rhizotron. 


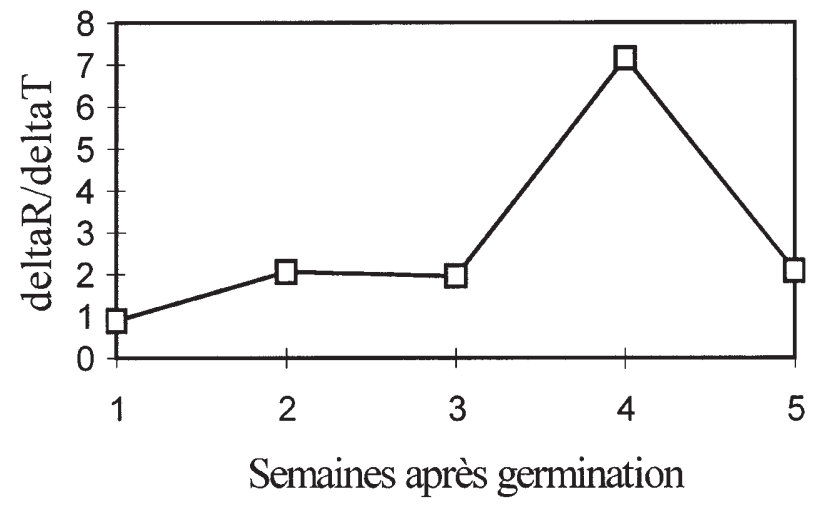

Figure 3. Ratio $\Delta \mathrm{R} / \Delta \mathrm{T}$ des accroissements hebdomadaires du pivot $(\Delta \mathrm{R})$ et de la tige principale $(\Delta \mathrm{T})$ de plantules d'A. africana élevées en rhizotron.

que la croissance caulinaire des plantules évolue vers une asymptote horizontale qui se situe aux environs de 25 centimètres. La croissance de la tige est continue sur une période de 4 à 5 semaines, suivie par une longue période de repos du bourgeon terminal. L'accroissement hebdomadaire de la tige principale a été du même ordre de grandeur que celui du pivot pendant la première semaine et plus faible durant les autres semaines (figure 3). Dans les deux premières semaines le système racinaire se résumait à un pivot glabre filiforme d'un millimètre de diamètre. Le pivot amorce ensuite un renflement progressif dans les dix premiers centimètres de sa partie supérieure. Sur cette partie se développe dès la troisième semaine un système racinaire secondaire dense et persistant. Trois mois après, le pivot présentait une forme conique bien nette. Le plus grand diamètre de la partie renflée attei- gnait $5 \mathrm{~mm}$ contre environ $2 \mathrm{~mm}$ pour le reste de la longueur du pivot. La vitesse de croissance des pivots a été variable d'une plantule à une autre et selon les horizons du sol. Elle a été en moyenne de $14,3 \mathrm{~cm}, 5,9 \mathrm{~cm}$ et $6,2 \mathrm{~cm}$ par semaine respectivement dans les horizons $\mathrm{H}_{1}$, $\mathrm{H}_{2}$ et $\mathrm{H}_{3}$ avec respectivement des écarts-types de 2,16, 2,52 et 2,68. La croissance des pivots 1 et 4 s'est estompée au seuil de l'horizon $\mathrm{H}_{4}$ tandis que celle des pivots 2 et 3 s'est poursuivie avec une vitesse de 3,9 et $7,3 \mathrm{~cm} /$ semaine (figure 4). La morphologie racinaire des plantules en milieu naturel était proche de celle observée en rhizotron. La croissance du pivot était ralentie ou déviée vers l'horizontale au contact des horizons argileux plus compacts. Les racines latérales, très superficielles, se sont essentiellement développées dans les premiers centimètres du sol et même dans les interstices de la litière en décomposition. Toutefois dans les conditions naturelles les racines secondaires se sont progressivement élaguées avec l'installation de la sécheresse. Un mois après la saison pluvieuse le système racinaire était réduit à un pivot conique relativement glabre.

La contribution du semis direct à la régénération d'A. africana dans la forêt est pratiquement nulle. Le parcours des transects dans les parcelles ensemencées de 1992 à 1996 n'a révélé la présence d'aucune plantule. Pourtant le taux de levée des graines après le semis semble être important. Les observations sur la parcelle nouvellement ensemencée montrent que deux mois après le semis le nombre de poquets ayant au moins une plantule était en moyenne de 7,84 poquets par placeau soit un taux de réussites de $87 \%$. Pendant la même période au total 460 plantules ont été recensées sur l'ensemble des 20 placeaux. Toutefois l'évolution démographique de ces plantules (figure 5) a montré une forte mortalité précoce. Un mois et demi après la saison pluvieuse la

Tableau II. Comparaison des accroissements moyens hebdomadaires du pivot et de la tige principale de plantules d'A. africana élevées en rhizotron.

\begin{tabular}{|c|c|c|c|c|c|c|c|c|c|c|c|c|}
\hline & \multicolumn{10}{|c|}{$\mathrm{n}^{\mathrm{e}}$ semaine après la germination } & \multirow[t]{2}{*}{$\sigma$} & \multirow[t]{2}{*}{ C.V. } \\
\hline & 1 & 2 & 3 & 4 & 5 & 6 & 7 & 8 & 9 & 10 & & \\
\hline $\begin{array}{l}\text { Accroissement moyen } \\
\text { hebdomadaire du pivot }(\Delta \mathrm{R})\end{array}$ & $14,38 \mathrm{a}$ & $4,63 \mathrm{~b}$ & $5,38 \mathrm{~b}$ & $6,38 \mathrm{~b}$ & $5,70 \mathrm{~b}$ & $6,68 \mathrm{~b}$ & $7,38 \mathrm{~b}$ & $5 \mathrm{~b}$ & $4,88 \mathrm{~b}$ & $3.5 \mathrm{~b}$ & 3,04 & $51,6 \%$ \\
\hline $\begin{array}{l}\text { Accroissement moyen } \\
\text { hebdomadaire de la tige } \\
\text { principale }(\Delta \mathrm{T})\end{array}$ & $16 \mathrm{a}$ & $2,25 \mathrm{~b}$ & $2,75 \mathrm{~b}$ & $0,88 \mathrm{~b}$ & $2,75 \mathrm{~b}$ & & & & & & 1,28 & $25,9 \%$ \\
\hline Ratio $\Delta \mathrm{R} / \Delta \mathrm{T}$ & 0,89 & 2,05 & 1,95 & 7,32 & 2,07 & & & & & & & \\
\hline
\end{tabular}

$\sigma=$ écart-type $; \mathrm{C} . \mathrm{V}=$ coefficient de variation.

NB : sur chaque ligne il n'y a pas de différence significative entre les accroissements suivis d'une même lettre au seuil de $5 \%$. 


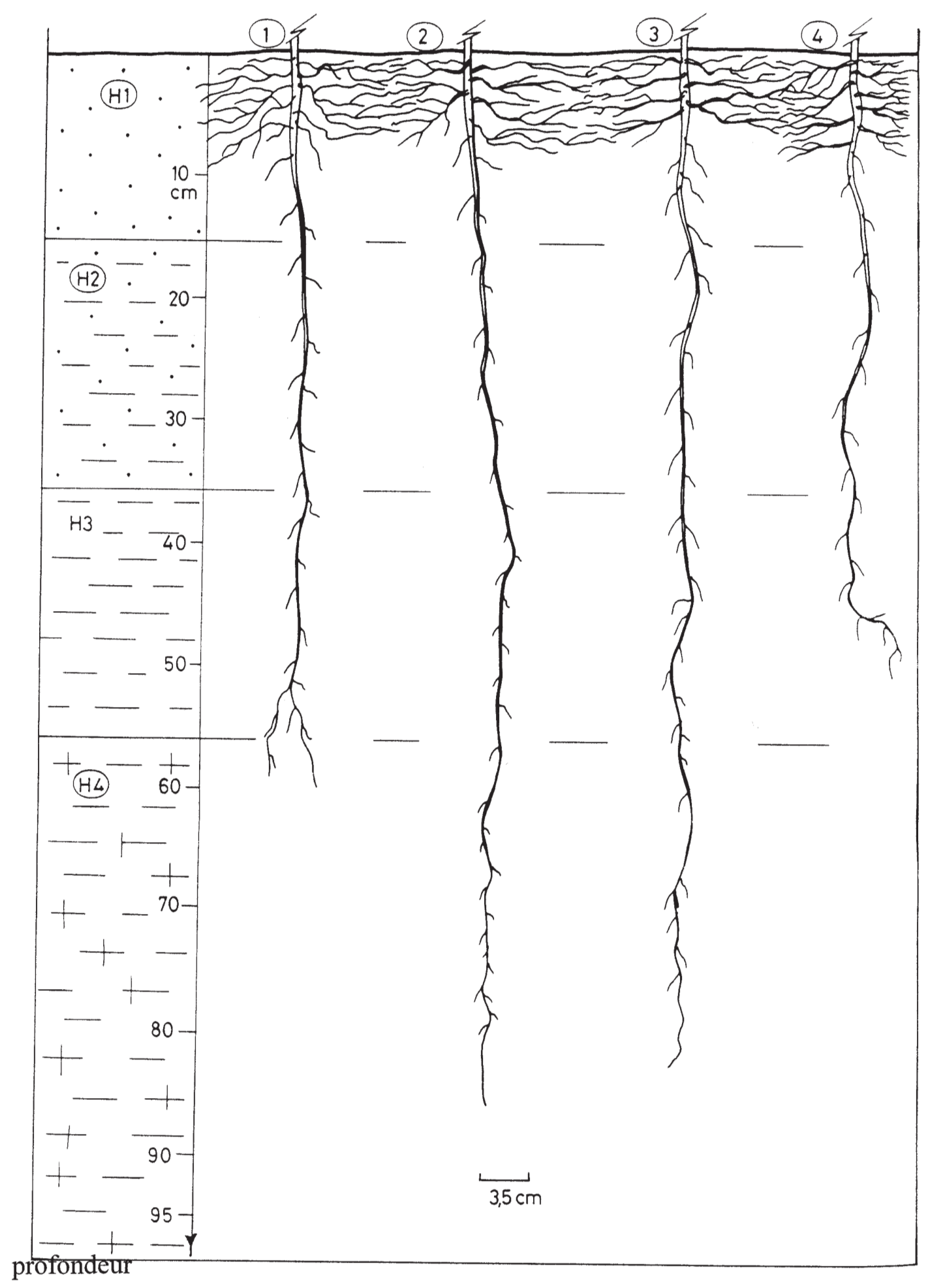

Figure 4. Morphologie racinaire de plantules d'Afzelia africana âgées de trois mois en rhizotron. 


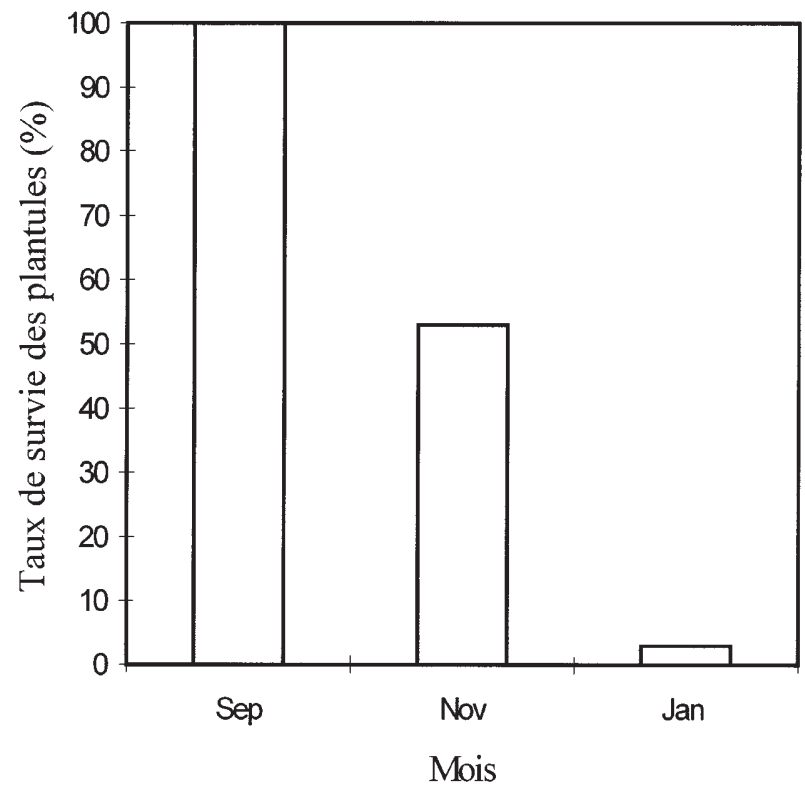

Figure 5. Évolution de la survie des plantules issues du semis directs d'Afzelia africana dans la forêt de Nazinon parcourue par les feux précoces.

mortalité des plantules atteignait $47 \%$ dont $15 \%$ par dessèchement de la plantule entière et le reste suite aux traumatismes de la partie aérienne dus principalement aux herbivores. Le dessèchement est plus fréquent chez les plantules installées dans les couches épaisses de litières où des nécroses des axes racinaires ont été observées. Un mois après les feux précoces, seules $3 \%$ des plantules, soit 14 plantules / 460, vivaient encore et s'observaient dans les zones non parcourues par les feux.

\section{DISCUSSION}

Un taux de germination de l'ordre de $80 \%$ après 33 mois de conservation dans les conditions ambiantes sans précaution particulière, traduit la bonne conservation des semences d'Afzelia africana. La teneur en eau exprimée en pourcentage du poids frais est sans doute l'un des principaux paramètres qui affectent la longévité des semences [23]. Une faible teneur en eau favorise une longue durée de conservation des semences. Selon Willan [23] une teneur en eau de 4 à $8 \%$ du poids frais est favorable à un entreposage des semences sans précautions particulières. Ces conditions s'opposent à celles des graines récalcitrantes qui ne peuvent subir une réduction importante de leur teneur en eau sans dommage pendant leur courte durée de vie. Les téguments relativement durs et imperméables des graines d'A. africana pourraient les protéger contre l'humidité ambiante qui occasionne parfois de fortes variations de la teneur en eau. Ces téguments induisent également une légère et différentielle dormance des graines, qui s'oppose à une germination groupée et homogène. Cela évite les fortes mortalités des plantules lorsque la germination in situ est suivie d'une période de sécheresse. L'échelonnement dans le temps de la levée des graines in situ est une stratégie d'adaptation des espèces à la forte variabilité de la pluviométrie [8].

La vigueur de la croissance aérienne dès la levée favorise l'adaptation des plantules à la concurrence herbacée. Cette adaptation est également favorisée par le système racinaire à la fois pivotant et traçant. Le développement et l'étalement précoce des racines secondaires permettent à la plantule d'exploiter un volume important de sol et d'accroître ainsi son absorption racinaire [19]. Cependant les plantules subissent une forte mortalité précoce. Celleci est due aux feux, au broutage par les herbivores, à la sécheresse et aux agents pathogènes tels que les invertébrés. Ces invertébrés sont sans doute parmi les principaux responsables des nécroses racinaires. La litière augmente en effet la population des invertébrés prédateurs des plantules [11]. Les termites particulièrement observés pourraient avoir un impact non négligeable. Leur rôle a été déjà évoqué dans les attaques racinaires de Balanites aegyptiaca [6]. Dans des cas similaires, Alexandre [1] a également mis en évidence la responsabilité des nématodes. Contrairement à d'autres principales espèces de la zone comme Detarium microcarpum [3] et Vittelaria paradoxa [10, 18], les graines d'Afzelia africana n'ont pas une germination cryptogée qui augmenterait la résistance des plantules aux feux et au broutage. Ses plantules ne sont pas non plus géophytes comme celles de Detarium microcarpum [3] qui se débarrassent de leurs parties aériennes pendant la saison sèche et conservent dans le sol leurs organes pérennants qui repoussent en saison pluvieuse. De plus elles ont un faible pouvoir de rejet, de sorte que la destruction de la partie aérienne par les feux ou par les animaux entraîne la mort chez la plupart de celles-ci. Le développement trop superficiel des racines a l'inconvénient de rendre les plantules particulièrement sensibles à la sécheresse et aux feux [16]. Il faut noter également le fait que les systèmes racinaires denses requièrent d'importantes quantités de carbone pour la production et le maintien des tissus [21]. Ce qui suggère la nécessité pour le développement des plantules d'A. africana d'un sol relativement fertile ayant un bilan hydrique favorable. L'élagage précoce des racines secondaires en milieu naturel semble traduire ces contraintes. La variation de la vitesse de croissance des pivots dans le rhizotron est en grande partie liée à l'hétérogénéité de la résistance à la pénétration du sol due à l'irrégularité du damage. Toutefois cet artefact révèle la forte sensibilité de la croissance racinaire à la compacité du sol. Cela est 
une contrainte majeure à l'installation et au développement des plantules d'A. africana dans la forêt classée de Nazinon où le sol est constamment piétiné par le bétail, et où la carapace ou la cuirasse se situe généralement à moins d'un mètre de profondeur [4].

\section{CONCLUSION}

Il existe une incompatibilité entre la pratique systématique des feux, la pâture et la pratique du semis direct d'A. africana. Les caractéristiques écophysiologiques et biologiques des plantules ne favorisent pas leur adaptation à ces facteurs. La régénération d'Afzelia africana dans les savanes nécessite une protection contre les feux et le pâturage. Dans la forêt classée de Nazinon où la pluviométrie dépasse rarement $1000 \mathrm{~mm} \mathrm{an}^{-1}$, Afzelia africana pourrait être proche de sa limite nord. De ce fait, son introduction devrait viser les zones plus humides telles que les dépressions et les zones ripicoles. L'étude de la durée de conservation sur la longévité des semences a révélé les possibilités de stockage des graines d'A. africana sans précautions particulières pendant trois saisons. Ces connaissances sont essentielles pour tendre progressivement vers une indépendance des sylviculteurs vis-à-vis des centres de commercialisation des semences forestières où le kilogramme de graines d'A. africana dépasse généralement $10000 \mathrm{CFA}$. Elles permettront une gestion plus rationnelle des semences très peu disponibles d'une part à cause de la faible représentativité de l'espèce et d'autre part, lié à l'émondage quasi-systématique des individus adultes par les éleveurs pour l'alimentation du bétail.

\section{RÉFÉRENCES}

[1] Alexandre D.Y., Régénération naturelle d'un arbre caractéristique de la forêt équatoriale de Côte d'Ivoire : Turraeanthus africana pelleger., Oecologia Plantarum, tome 12 (1977) 241-262.

[2] Bariteau M., Régénération naturelle de la forêt tropicale humide de Guyane : étude de la répartition spatiale de Qualea rosa Aublet, Eperna falcata Aublet et Symphonia globulifera Linnaeus f., Ann. Sci. For. 49 (1992) 359-392.

[3] Bationo B.A., Étude de la régénération séminale des ligneux dans les jachères de Sobaka, mémoire de DEA, Université de Ouagadougou, 1996, 62 p.

[4] De Blic Ph., Somé N.A., État structural d'horizons superficiels sableux sous culture ou jachère herbacée en Afrique de l'ouest (Burkina Faso), Étude et gestion des sols 4 (1997) 17-24.

[5] Dirik H., Effet du stress hydrique osmotique sur la germination des graines chez les provenances de Cèdre du Liban (Cedrus Libani A. Rich) d'origine turque, Ann. For. Sci. 57 (2000) 361-367

[6] Ganaba S., Rôle des structures racinaires dans la dynamique du peuplement ligneux de la région de la Mare d'Oursi entre 1980 et 1992 , thèse de doctorat, Université de Ouagadougou, 1994.

[7] Gorse V., Reproduction sexuée et hétérogénéité spatiale des semis chez quelques arbres des savanes arborées du Nazinon (Burkina Faso), O.R.S.T.O.M., 1994, 37 p.

[8] Grouzis M., Structure, productivité et dynamique des systèmes écologiques sahéliens (Mare d'Oursi, Burkina faso), O.R.S.T.O.M., 1978.

[9] Guinko S., La végétation de la Haute-volta. Tome1, thèse de doctorat, Bordeaux 3, 1984, 318 p.

[10] Jackson G., Cryptogeal germination and other seedling adaptation to the buring of vegetation in savanna region in the origin of pyrophytic habit, New phytol. 73 (1974) 771-780.

[11] José M.J., Multiple indirect effects of plant litter affect the establishement of woody seedlings in old fields, Ecology 75 (1994) 1727-1735.

[12] Jurado E., Westoby M., Seedling growth in relation to size among species of arid australia, J. Ecol. 80 (1992) 407-416.

[13] Lopez M., Humara J.M., Casares A., Majada J., The effect of temperature and water stress on laboratory germination of Eucalpytus globulus Labill. seeds of different sizes, Ann. For. Sci. 57 (2000) 245-250.

[14] Mapongmetsem P.M., Duguma B., Nkongmeneck B.A., Selegny E., The effect of various seed pretreatments to improve germination in eight indigenous tree species in the forest of Cameroon, Ann. For. Sci. 56 (1999) 679-684.

[15] Miquel M., Morphologie fonctionnelle de plantules d'espèces forestières du Gabon, Bull. Mus. Hist. Nat., Paris, 4 e sér. 9 (1987) 101-121.

[16] Misra D.K., Relation of root development to drougth resistance of plants, Kansas State college, USA, 1954.

[17] Orozco-Segovia A., Vazquez-Yanes C., Effect of moisture on longevity in seeds of some rain forest species, Biotropica 22 (1993) 215-216.

[18] Ouedraogo J.S., Alexandre D.Y., Distribution des principales espèces agroforestières à Watinoma, terroir du plateau central burkinabè, une résultante de contraintes écologiques et antropiques, J. Agric. Trad. Bot. Appl. XXXVI (1994) $101-111$.

[19] Rabesandratana R.N., Évaluation de la biomasse d'Euphorbia stenoclata Bill. (Euphorbiaceae) dans la région de Tuléar (sud-ouest de Madagascar), Sécheresse 10 (1999) $55-61$.

[20] Sambou B., Goudiaby A., Madsen J.E., Bâ A.T., Étude comparative des modification de la flore et de la végétation ligneuse dans les forêts classées de Koutal et de l'île Kouyong (centre-ouest du Sénégal), J. Agric. Trad. Bot. Appl. XXXVI (1994) 87-100.

[21] Smucker A.J.M., Aiken R.M., Dynamic root responses to water deficits, Soil Sci. 154 (1992) 281-289.

[22] Vetaas O.R., Microsites effects of tree and shrubs in dry savannas, J. Végét. Sci. 3 (1992) 337-344.

[23] Willan R.L., Guide de manipulation des semences forestières dans le cas particulier des régions tropicales, étude FAO Forêts 20/2, 1992. 